\title{
Finite Difference Analysis of Radiative Free Convection Flow Past an Impulsively Started Vertical Plate with Variable Heat and Mass Flux
}

\author{
V.R. Prasad ${ }^{1}$, N. Bhaskar Reddy ${ }^{2}$, R. Muthucumaraswamy ${ }^{3}$ and B.Vasu ${ }^{4}$ \\ ${ }^{1}$ Department of Mathematics, Madanapalle Institute of Technology and Science, Madanapalle-517325. \\ ${ }^{2}$ Department of Mathematics, Sri Venkateswara University, Tirupati-517502, India. \\ ${ }^{3}$ Department of Information Technology,Sri Venkateswara College of Engineering, Sriperumbudur- 602105, India. \\ ${ }^{4}$ Department of Mathematics, Sri Venkateswara University, Tirupati-517502, India.
}

Email:rcpmaths@mits.ac.in

(Received February 25, 2009; accepted September 21, 2009)

\begin{abstract}
A numerical solution of the unsteady radiative free convection flow of an incompressible viscous fluid past an impulsively started vertical plate with variable heat and mass flux is presented here. This type of problem finds application in many technological and engineering fields such as rocket propulsion systems, spacecraft re-entry aerothermodynamics, cosmical flight aerodynamics, plasma physics, glass production and furnace engineering. The fluid is gray, absorbing-emitting but non-scattering medium and the Rosseland approximation is used to describe the radiative heat flux in the energy equation. The governing non-linear, coupled equations are solved using an implicit finite difference scheme. Numerical results for the velocity, temperature, concentration, the local and average skinfriction, the Nusselt and Sherwood number are shown graphically, for different values of Prandtl number, Schmidt number, thermal Grashof number, mass Grashof number, radiation parameter, heat flux exponent and the mass flux exponent. It is observed that, when the radiation parameter increases, the velocity and temperature decrease in the boundary layer. The local and average skin-friction increases with the increase in radiation parameter. For increasing values of radiation parameter the local as well as average Nusselt number increases.
\end{abstract}

Keywords: Finite difference scheme, variable heat and mass flux, radiation.

\begin{tabular}{|c|c|}
\hline $\mathrm{a}, \mathrm{b}$ & constants \\
\hline $\mathrm{Cp}$ & specific heat \\
\hline$C^{\prime}$ & concentration \\
\hline $\mathrm{C}$ & dimensionless concentration \\
\hline $\mathrm{D}$ & mass diffusion coefficient \\
\hline $\mathrm{g}$ & acceleration due to gravity \\
\hline $\mathrm{Gm}$ & mass Grashof number \\
\hline Gr & thermal Grashof number \\
\hline $\mathrm{k}$ & thermal conductivity \\
\hline $\mathrm{L}$ & reference length \\
\hline $\mathrm{m}$ & $\begin{array}{l}\text { exponent in the power law variation of } \\
\text { heat flux }\end{array}$ \\
\hline $\mathrm{n}$ & $\begin{array}{l}\text { exponent in the power law variation mass } \\
\text { flux }\end{array}$ \\
\hline $\mathrm{N}$ & conduction-radiation parameter \\
\hline${ }^{N} u_{X}$ & dimensionless local Nusselt number \\
\hline$\overline{\mathrm{Nu}}$ & dimensionless average Nusselt number \\
\hline $\operatorname{Pr}$ & Prandtl number \\
\hline$q_{w}^{\prime}$ & heat flux per unit area at the plate \\
\hline$q_{w}^{*}$ & mass flux per unit area at the plate \\
\hline
\end{tabular}

\begin{tabular}{|c|c|c|}
\hline \multicolumn{3}{|c|}{ NOMENCLATURE } \\
\hline & $\mathrm{Sc}$ & Schmidt number \\
\hline & $S h_{X}$ & dimensionless local Sherwood number \\
\hline & $\overline{S h}$ & dimensionless average Sherwood number \\
\hline & $T^{\prime}$ & temperature \\
\hline & $\mathrm{T}$ & dimensionless temperature \\
\hline & $t^{\prime}$ & time \\
\hline & $\mathrm{t}$ & dimensionless time \\
\hline & $\mathrm{u}_{0}$ & velocity of the plate \\
\hline & $\mathrm{u}, \mathrm{v}$ & $\begin{array}{l}\text { velocity components in } \mathrm{x}, \mathrm{y} \text { - directions } \\
\text { respectively }\end{array}$ \\
\hline nass & $\mathrm{U}, \mathrm{V}$ & $\begin{array}{l}\text { dimensionless velocity components in } \\
\mathrm{X}, \mathrm{Y} \text { directions respectively }\end{array}$ \\
\hline & $\mathrm{X}$ & $\begin{array}{l}\text { dimensionless spatial coordinate along } \\
\text { the plate }\end{array}$ \\
\hline & $\mathrm{y}$ & spatial coordinate normal to the plate \\
\hline & $\mathrm{Y}$ & $\begin{array}{l}\text { dimensionless spatial coordinate normal to } \\
\text { the plate }\end{array}$ \\
\hline & Greek s & mbols \\
\hline & $\alpha$ & thermal diffusivity \\
\hline & $\beta$ & volumetric coefficient of thermal expansion \\
\hline
\end{tabular}




\author{
$\beta^{*} \quad$ volumetric coefficient of expansion with \\ concentration \\ $v \quad$ kinematic viscosity \\ $\rho \quad$ density \\ $\tau_{X} \quad$ dimensionless local skin-friction
}

\section{INTRODUCTION}

Numerical modeling of combined heat and mass transfer flows is important in many industrial applications including geothermal energy, solar energy systems, biomechanics [Wang and Chen (2005)] and rocket propulsion [Vrentas and Vrentas (1996)]. Extensive studies have been reported in all these areas employing a wide variety of numerical simulation methods including finite elements, finite differences, network simulation and CFD (Computational Fluid Dynamics). Many high temperature processes in industrial design and combustion and fire science involve thermal radiation heat transfer in combination with conduction, convection and also mass transfer. For example radiative-convective heat transfer flows arise in industrial furnace systems, radiative waste storage, forest fire dynamics, fire spread in buildings etc. Considerable research has therefore appeared studying radiative-convective flows in a variety of geometrical configurations with numerical and mathematical models. Bratis and Novotny (1974) reported on the effects of thermal radiation in the convection boundary layer regime of an enclosure. Chang et al. (1983) used a radiative flux diffusion approximation to model the interaction of convective and radiative heat transfer in two-dimensional complex enclosure. Hossain and Vafai (2001) reported on the natural convection boundary layer heat transfer with variable viscosity, suction and radiation effects. The interaction of radiation with laminar free convection heat transfer from a vertical plate was investigated by Cess (1966) for an absorbing, emitting fluid in the optically thick region, using the singular perturbation technique. Chamkha et al. (2001) studied computationally the influence of mass transfer and radiation flux on natural convection flows. Cheng and Ozisik (1972) studied a related problem for an absorbing, emitting and isotropically scattering fluid and treated the radiation part of the problem exactly with the normal mode expansion technique. The unsteady flow past a moving plate in the presence of free convection and radiation was studied by Mansour (1990). The unsteady flow past a moving plate in the presence of free convection and radiation was studied by Raptis and Perdikis (1999). Das et al. (1996) analyzed the radiation effects on flow past an impulsively started infinite isothermal vertical plate. Abd-El-Naby et al. (2003) studied the effects of radiation on unsteady free convective flow past a semiinfinite vertical plate with a variable surface temperature. Mukhopadhyay (2009) studied the effects of radiation and variable fluid viscosity on flow and heat transfer along a symmetric wedge. Chamkha et al. (2004) used the Rosseland diffusion flux model to analyze the buoyancy-driven dissipative natural convection-radiation boundary layer flow from a wedge in a porous medium. They showed that an increase in Boltzmann-Rosseland radiation-conduction number and negative Eckert number enhances heat transfer $\bar{\tau} \quad$ dimensionless average skin-friction

\begin{tabular}{|c|c|}
\hline Sul & ipts \\
\hline w & onditions on the wal \\
\hline$\infty$ & free stream condition \\
\hline
\end{tabular}

gradients at the wedge face considerably. Recently Prasad et al. (2007) studied radiation and mass transfer effects on two-dimensional flow past an impulsively started infinite vertical plate

In certain industrial systems, the flow past an impulsively started plate is also important. Such flows are transient and therefore temporal velocity and temperature gradients have to be included in the analysis. Excellent work in this regard has been presented by Stewartson (1951) although his study ignored heat transfer. Later Soundalgekar (1977) extended this study obtaining Laplace transform solutions for the natural convection and mass transfer effects on flow past impulsively started vertical surface. More recently Muthucumaraswamy and Ganesan (1998) considered transient heat and mass transfer past an impulsively started vertical plate.

The aim of the present paper is to study an unsteady two-dimensional laminar free convective flow of a viscous, incompressible fluid past an impulsively started moving plate with variable heat and mass flux, in the presence of thermal radiation effects. The equations of continuity, linear momentum, energy and species concentration, which govern the flow field are solved by using an implicit finite difference scheme of Crank-Nicolson type.

\section{MATHEMATICAL ANALYSIS}

The basic equations, used to interpret and analyze natural convection, are the partial differential equations, which result from the consideration of the conservation of mass, Navier-Stokes equation along $\mathrm{x}$-direction and $y$-direction, energy and mass diffusion equation. In free convection, the fluid motion arises solely from the buoyancy forces. The buoyancy effect arises due to the interaction between density differences in a body of fluid and a body force, usually gravitational force. The density differences are due to the temperature differences or concentration differences of the diffusing species or the combination of both. So, both thermal and mass diffusing processes must be considered simultaneously for all aspects of the flow.

Consider the laminar time dependent viscous buoyancy driven heat and mass transfer in an incompressible, absorbing emitting and non-scattering gray Newtonian fluid past an impulsively started vertical surface. The $X^{\prime}$ direction is located parallel to the plate surface and the $Y^{\prime}$ direction normal to it. At time $t^{\prime}=0$, the plate commences impulsive motion in the $X^{\prime}$ direction with constant velocity $u_{0}^{\prime}$. Initially, it is assumed that the plate and the fluid are at the same temperature $T_{\infty}^{\prime}$ and concentration level $C_{\infty}^{\prime}$ everywhere in the fluid. At time $t^{\prime}>0$, the plate starts moving impulsively in the 
vertical direction with constant velocity $\mathrm{u}_{0}$ against the gravitational field. Also, it is assumed that the heat and mass is supplied from the plate to the fluid at a rate of $q_{w}^{\prime}(x)=a x^{m}$ and $q_{w}^{*}(x)=b x^{n} \quad$ respectively, and they are maintained at the same level. It is also assumed that the heat due to viscous dissipation in the energy equation is very small and is neglected. It is also assumed that the concentration of the diffusing species in the binary mixture is very less in comparison to the other chemical species, which are present and hence the Soret and Dufour effects are negligible. The boundary layer equations for mass, momentum, heat and species conservation in an $(x, y)$ coordinate system can be shown to take the form:

$$
\begin{aligned}
& \frac{\partial u}{\partial x}+\frac{\partial v}{\partial y}=0 \\
& \rho\left(\frac{\partial u}{\partial t^{\prime}}+u \frac{\partial u}{\partial x}+v \frac{\partial u}{\partial y}\right)=-\frac{\partial p}{\partial x}-\rho g \beta+\mu \frac{\partial^{2} u}{\partial y^{2}} \\
& \frac{\partial T^{\prime}}{\partial t^{\prime}}+u \frac{\partial T^{\prime}}{\partial x}+v \frac{\partial T^{\prime}}{\partial y}=\alpha \frac{\partial^{2} T^{\prime}}{\partial y^{2}}-\frac{1}{\rho c_{p}} \frac{\partial q_{r}}{\partial y} \\
& \frac{\partial C^{\prime}}{\partial t^{\prime}}+u \frac{\partial C^{\prime}}{\partial x}+v \frac{\partial C^{\prime}}{\partial y}=D \frac{\partial^{2} C^{\prime}}{\partial y^{2}}
\end{aligned}
$$

The fluid properties are assumed to be constant except for the density in the body force term. The unheated fluid far removed from the plate is in hydrostatic equilibrium, or $\left(\partial p_{e} / \partial x\right)=-\rho_{e} g$, where the subscript $e$ denotes equilibrium conditions. At any elevation, the pressure is uniform and therefore $\partial p / \partial x=\partial p_{e} / \partial x$. Substituting $\rho_{e} g$ for $-(\partial p / \partial x)$ in Eq. (2) gives

$$
\rho\left(\frac{\partial u}{\partial t^{\prime}}+u \frac{\partial u}{\partial x}+v \frac{\partial u}{\partial y}\right)=-\left(\rho_{e}-\rho\right) g+\mu \frac{\partial^{2} u}{\partial y^{2}}
$$

The further simplification can be made by assuming that the density $\rho$ depends only on the temperature and not on the pressure. For an incompressible fluid this is self evident, but for a gas it implies that the vertical dimension of the body is small enough that the hydrostatic density $\rho_{e}$ is constant. This simplification is referred to as Boussinesq approximation. Since the flow is driven by the buoyancy forces arising from the density differences due to both temperature and concentration difference, with these assumptions buoyant term can be written as

$$
\begin{aligned}
g\left(\rho_{e}-\rho\right)= & g\left(\rho_{\infty}-\rho\right)=-g \rho \beta\left(T_{\infty}^{\prime}-T^{\prime}\right) \\
& -g \rho \beta^{*}\left(C_{\infty}^{\prime}-C^{\prime}\right)
\end{aligned}
$$

Where $\beta$ is the volumetric coefficient of thermal expansion, $\beta^{*}$ is the coefficient of volumetric expansion with concentration and is defined as

$$
\begin{aligned}
& \beta=\left(-\frac{1}{\rho} \frac{\partial \rho}{\partial T^{\prime}}\right)_{p} \cong \frac{\rho_{\infty}-\rho}{\rho\left(T^{\prime}-T_{\infty}^{\prime}\right)}, \\
& \beta^{*}=\left(-\frac{1}{\rho} \frac{\partial \rho}{\partial C}\right)_{p} \cong \frac{\rho_{\infty}-\rho}{\rho\left(C-C_{\infty}\right)}
\end{aligned}
$$

For an ideal gas (i.e. $\rho=p / R T$ ) the coefficient of thermal expansion is

$\beta=\frac{1}{T_{\infty}^{\prime}}, \beta^{*}=\frac{1}{C_{\infty}^{\prime}}$

where the temperature $T_{\infty}$ and $C_{\infty}$ are absolute temperature and concentration far from the plate .

The equation of motion for natural convection is obtained by substituting the buoyant term, Eq. (6), into Eq. (5), yielding

$$
u \frac{\partial u}{\partial x}+v \frac{\partial u}{\partial y}=g \beta\left(T^{\prime}-T_{\infty}^{\prime}\right)+g \beta^{*}\left(C^{\prime}-C_{\infty}^{\prime}\right)+v \frac{\partial^{2} u}{\partial y^{2}}
$$

The initial and boundary conditions are

$t^{\prime} \leq 0: u=0, \quad v=0, T^{\prime}=T_{\infty}^{\prime}, \quad C^{\prime}=C_{\infty}^{\prime}$

$$
\begin{aligned}
t^{\prime}>0: u=u_{0}, v=0, \frac{\partial T^{\prime}}{\partial y} & =-\frac{q_{w}^{\prime}(x)}{k}, \\
\frac{\partial C^{\prime}}{\partial y} & =-\frac{q_{w}^{*}(x)}{D} \text { at } y=0
\end{aligned}
$$$$
u=0, T^{\prime}=T_{\infty}^{\prime}, C^{\prime}=C_{\infty}^{\prime} \text { at } x=0
$$

$u \rightarrow 0, T^{\prime} \rightarrow T_{\infty}^{\prime}, C^{\prime} \rightarrow C_{\infty}^{\prime}$ as $\quad \mathrm{y} \rightarrow \infty$

where $\mathrm{x}$ and $\mathrm{y}$ are coordinates, $u, v$ are the velocity components in the $\mathrm{x}, \mathrm{y}$ directions, $t^{\prime}$ is dimensionless time, $\mathrm{g}$ is the gravitational acceleration, $\beta$ is the coefficient of thermal expansion, $\beta^{*}$ is the mass transfer coefficient of expansion, $v$ is the kinematic viscosity of the gray fluid, $T^{\prime}$ is temperature, $C^{\prime}$ is species concentration, $\alpha$ is the thermal diffusivity, $\mathrm{D}$ is the species diffusivity, ()$_{w}$ denotes conditions at the wall, ()$_{\infty}$ designates the condition in the free stream(outside the boundary layers).

Employing the Rosseland diffusion approximation, Modest (1993) leads to the following expression for radiative heat flux $q_{r}$ in the energy conservation Eq. (3):

$$
q_{r}=-\frac{4 \sigma_{S}}{3 k_{e}} \frac{\partial T^{\prime}}{\partial y}
$$

where $\sigma_{s}$ is the Stefan-Boltzmann constant and $k_{e}$ is the mean absorption coefficient, respectively. It should be noted that by using the Rosseland approximation we limit our analysis to optically thick fluids. Refractive index of the gas medium is constant. Unidirectional radiation flux, $q_{r}$ is considered and it is assumed that $\frac{\partial q_{r}}{\partial y} \gg \frac{\partial q_{r}}{\partial x}$. This model is valid for optically-thick media in which thermal radiation propagates only a limited distance prior to experiencing scattering or absorption. The local thermal radiation intensity is due to radiation emanating from proximate locations in the vicinity of which emission and scattering are comparable to the location of interest. The energy transfer depends on conditions only in the area adjacent to the plate regime i.e. the boundary layer regime. Rosseland's model yields accurate results for intensive absorption i.e. optically-thick flows which are optically 
far from the bounding surface. Implicit in this approximation is also the existence of wavelength regions where the optical thickness may exceed a value of five. As such the Rosseland model, while limited compared with other flux models, can simulate to a reasonable degree of accuracy thermal radiation in problems ranging from thermal radiation transport via gases at low density to thermal radiation simulations associated with nuclear blast waves. If temperature differences within the flow are sufficiently small, then Eq. (10) can be linearized by expanding $T^{4}$ into the Taylor series about $T_{\infty}^{\prime}$, which after neglecting higher order terms takes the form:

$T^{\prime 4} \cong 4 T_{\infty}^{\prime 3} T^{\prime}-3 T_{\infty}^{\prime 4}$

In view of Eqs. (10) and (11), Eq.(3) reduces to

$\frac{\partial T^{\prime}}{\partial t^{\prime}}+u \frac{\partial T^{\prime}}{\partial x}+v \frac{\partial T^{\prime}}{\partial y}=\alpha \frac{\partial^{2} T^{\prime}}{\partial y^{2}}+\frac{16 \sigma_{s} T_{\infty}^{\prime 3}}{3 k_{e} \rho c_{p}} \frac{\partial^{2} T^{\prime}}{\partial y^{2}}$

Local and average skin-friction are given respectively by

$$
\begin{aligned}
& \tau_{x}^{\prime}=-\mu\left(\frac{\partial u}{\partial y}\right)_{y=0} \\
& \overline{\tau_{L}}=\frac{-1}{L} \int_{0}^{L} \mu\left(\frac{\partial u}{\partial y}\right)_{y=0} d x
\end{aligned}
$$

Local and average Nusselt number are given respectively by

$$
\begin{aligned}
N u_{x}=\frac{-x\left(\frac{\partial T^{\prime}}{\partial y}\right)_{y=0}}{T_{w}^{\prime}-T_{\infty}^{\prime}} \\
\overline{N u}_{L}=-\int_{0}^{L}\left[\left(\frac{\partial T^{\prime}}{\partial y}\right)_{y=0} /\left(T_{w}^{\prime}-T_{\infty}^{\prime}\right)\right] d x
\end{aligned}
$$

Local and average Sherwood number are given respectively by

$$
\begin{aligned}
S h_{x} & =\frac{-x\left(\frac{\partial C^{\prime}}{\partial y}\right)_{y=0}}{C_{w}^{\prime}-C_{\infty}^{\prime}} \\
\overline{S h}_{L} & =-\int_{0}^{L}\left[\left(\frac{\partial C^{\prime}}{\partial y}\right)_{y=0} /\left(C_{w}^{\prime}-C_{\infty}^{\prime}\right)\right] d x
\end{aligned}
$$

On introducing the following non-dimensional quantities

$$
\begin{aligned}
& X=\frac{x u_{0}}{L}, \quad Y=\frac{y \sqrt{u_{0}}}{\sqrt{L v}}, t=\frac{t^{\prime} u_{0}}{L}, U=\frac{u}{u_{0}}, \\
& V=\frac{v \sqrt{L}}{\sqrt{u_{0} v}}, G m=\frac{\operatorname{Lg} \beta^{*}\left(\frac{q_{w}^{*}(L) \sqrt{L v}}{D \sqrt{u_{0}}}\right)}{u_{0}^{2}}, \\
& G r=\frac{\operatorname{Lg} \beta\left(\frac{q_{w}^{\prime}(L) \sqrt{L v}}{k \sqrt{u_{0}}}\right)}{u_{0}^{2}}, N=\frac{k_{e} k}{4 \sigma_{s} T_{\infty}^{\prime 3}}
\end{aligned}
$$

$$
\begin{aligned}
& T=\frac{T^{\prime}-T_{\infty}^{\prime}}{\left(\frac{q_{w}^{\prime}(L) \sqrt{L v}}{k \sqrt{u_{0}}}\right)} \quad C=\frac{C^{\prime}-C_{\infty}^{\prime}}{\left(\frac{q_{w}^{*}(L) \sqrt{L v}}{D \sqrt{u_{0}}}\right)}, \\
& \operatorname{Pr}=\frac{v}{\alpha}, S c=\frac{v}{D}
\end{aligned}
$$

Equations (1), (2), (12) and (4) are reduced to the following non-dimensional form

$$
\begin{aligned}
& \frac{\partial U}{\partial X}+\frac{\partial V}{\partial Y}=0 \\
& \frac{\partial U}{\partial t}+U \frac{\partial U}{\partial X}+V \frac{\partial U}{\partial Y}=G r T+G m C+\frac{\partial^{2} U}{\partial Y^{2}} \\
& \frac{\partial T}{\partial t}+U \frac{\partial T}{\partial X}+V \frac{\partial T}{\partial Y}=\frac{1}{\operatorname{Pr}}\left(1+\frac{4}{3 N}\right) \frac{\partial^{2} T}{\partial Y^{2}} \\
& \frac{\partial C}{\partial t}+U \frac{\partial C}{\partial X}+V \frac{\partial C}{\partial Y}=\frac{1}{S c} \frac{\partial^{2} C}{\partial Y^{2}}
\end{aligned}
$$

Where $\mathrm{X}$ and $\mathrm{Y}$ are dimensionless coordinates, $\mathrm{U}$ and $\mathrm{V}$ are dimensionless velocities, $\mathrm{t}$ is the dimensionless time, $\mathrm{T}$ is the dimensionless temperature function, $\mathrm{C}$ is dimensionless concentration function, $\mathrm{N}$ is the conduction-radiation heat transfer parameter, $\operatorname{Pr}$ is Prandtl number, Sc is Schmidt number, Gr is thermal Grashof number, $\mathrm{Gm}$ is species Grashof number.

The corresponding initial and boundary conditions are $t \leq 0: U=0, V=0, T=0, C=0$

$$
\begin{aligned}
& t>0: U=1, V=0, \frac{\partial T}{\partial Y}=-X^{m}, \frac{\partial C}{\partial Y}=-X^{n} \text { at } Y=0 \\
& U=0, T=0, C=0 \text { at } X=0 \\
& U \rightarrow 0, T \rightarrow 0, C \rightarrow 0 \text { as } Y \rightarrow \infty
\end{aligned}
$$

Local and average skin-friction in non-dimensional form are

$$
\begin{aligned}
& \tau_{X}=\frac{\tau^{\prime}}{\rho u_{0}^{2}}=-\left(\frac{\partial U}{\partial Y}\right)_{Y=0} \\
& \bar{\tau}=-\int_{0}^{1}\left(\frac{\partial U}{\partial Y}\right)_{Y=0} d X
\end{aligned}
$$

Local and average Nusselt number in non-dimensional form are

$$
\begin{aligned}
& N u_{X}=-X\left[\left(\frac{\partial T}{\partial Y}\right)_{Y=0} / T_{Y=0}\right] \\
& \overline{N u}=-\int_{0}^{1}\left[\left(\frac{\partial T}{\partial Y}\right)_{Y=0} / T_{Y=0}\right] d X
\end{aligned}
$$

Local and average Sherwood number in nondimensional form are

$$
\begin{aligned}
& S h_{X}=-X\left[\left(\frac{\partial C}{\partial Y}\right)_{Y=0} / C_{Y=0}\right] \\
& \overline{S h}=-\int_{0}^{1}\left[\left(\frac{\partial C}{\partial Y}\right)_{Y=0} / C_{Y=0}\right] d X
\end{aligned}
$$

\section{NUMERICAL TECHNIQUE}

In order to solve these unsteady, non-linear coupled equations (20) to (23) under the conditions (24), an implicit finite difference scheme of Crank-Nicolson type has been employed. This method has been 
extensively developed in recent years and remains one of the most reliable procedures for solving partial differential equation systems. It is unconditionally stable. It utilizes a central differencing procedure for space and is an implicit method. The partial differential terms are converted to difference equations and the resulting algebraic problem is solved using a triadiagonal matrix algorithm. For transient problems a trapezoidal rule is utilized and provides second-order convergence. The Crank-Nicolson Method (CNM) scheme has been used in numerous heat transfer, radiation and convection flow problems. Ransom and Fulton (1985) discussed a modified, optimized version of the Crank-Nicolson method for general thermal engineering problems. Lin and Huang (1991) studied thermal radiation effects on laminar forced convection in thermally developing circular pipe flow using an integral formulation for the divergence of radiative heat flux, a finite element node approximation technique and the Crank-Nicolson finite difference method with an iterative procedure. Hadley (1992) used the Crank-Nicolson method with a new boundary condition to study beam propagation emitting radiation with a minimum reflection coefficient, considering both two and three-dimensional cases. Krishnaprakas et al. (2001) used the Chandrasekhar discrete ordinates method and the Crank-Nicolson method to study combined conduction and radiation heat transfer in a gray planar nonlinearly anisotropic scattering medium bounded between two plane parallel surfaces reflecting both diffusely and specularly. Prasad et al. (2007) used Crank-Nicolson scheme to analyze the transient convective heat and mass transfer with thermal radiation effects along a vertical impulsively started plate. The CNM method has been found to work very efficiently for parabolic type partial differential equations as exemplified by boundary-layer flows The finite difference equations corresponding to equations (20) to (23) are discretized using CNM as follows: Mass Conservation:

$$
\begin{aligned}
& \frac{\left[U_{i, j}^{n+1}-U_{i-1, j}^{n+1}+U_{i, j}^{n}-U_{i-1, j}^{n}+U_{i, j-1}^{n+1}-U_{i-1, j-1}^{n+1}+U_{i, j-1}^{n}-U_{i-1, j-1}^{n}\right]}{4 \Delta X} \\
& +\frac{\left[V_{i, j}^{n+1}-V_{i, j-1}^{n+1}+V_{i, j}^{n}-V_{i, j-1}^{n}\right]}{2 \Delta Y}=0
\end{aligned}
$$

Momentum Conservation:

$$
\begin{aligned}
& \frac{\left[U_{i, j}^{n+1}-U_{i, j}^{n}\right]}{\Delta t}+U_{i, j}^{n} \frac{\left[U_{i, j}^{n+1}-U_{i-1, j}^{n+1}+U_{i, j}^{n}-U_{i-1, j}^{n}\right]}{2 \Delta X} \\
& +V_{i, j}^{n} \frac{\left[U_{i, j+1}^{n+1}-U_{i, j-1}^{n+1}+U_{i, j+1}^{n}-U_{i, j-1}^{n}\right]}{4 \Delta Y} \\
& =G r \frac{\left[T_{i, j}^{n+1}+T_{i, j}^{n}\right]}{2}+G m \frac{\left[C_{i, j}^{n+1}+C_{i, j}^{n}\right]}{2} \\
& +\frac{\left[U_{i, j-1}^{n+1}-2 U_{i, j}^{n+1}+U_{i, j+1}^{n+1}+U_{i, j+1}^{n+1}-2 U_{i, j}^{n}+U_{i, j+1}^{n}\right]}{2(\Delta Y)^{2}}
\end{aligned}
$$

Energy (Heat) Conservation:

$$
\begin{aligned}
& \frac{\left[T_{i, j}^{n+1}-T_{i, j}^{n}\right]}{\Delta t}+U_{i, j}^{n} \frac{\left[T_{i, j}^{n+1}-T_{i-1, j}^{n+1}+T_{i, j}^{n}-T_{i-1, j}^{n}\right]}{2 \Delta X} \\
& +V_{i, j}^{n} \frac{\left[T_{i, j}-T_{i, j-1}^{n+1}+T_{i, j+1}^{n}-T_{i, j-1}^{n}\right]}{4 \Delta Y}
\end{aligned}
$$

$$
=\frac{1}{\operatorname{Pr}}\left(1+\frac{4}{3 N}\right) \frac{\left[T_{i, j-1}^{n+1}-2 T_{i, j}^{n+1}+T_{i, j+1}^{n+1}+T_{i, j-1}^{n}-2 T_{i, j}^{n}+T_{i, j+1}^{n}\right]}{2(\Delta Y)^{2}}
$$

Species (Concentration) Conservation:

$\frac{\left[C_{i, j}^{n+1}-C_{i, j}^{n}\right]}{\Delta t}+U_{i, j}^{n} \frac{\left[C_{i, j}^{n+1}-C_{i-1, j}^{n+1}+C_{i, j}^{n}-C_{i-1, j}^{n}\right]}{2 \Delta X}$

$+V_{i, j}^{n} \frac{\left[C_{i, j+1}^{n+1}-C_{i, j-1}^{n+1}+C_{i, j+1}^{n}-C_{i, j-1}^{n}\right]}{4 \Delta Y}$

$=\frac{1}{S c} \frac{\left[C_{i, j-1}^{n+1}-2 C_{i, j}^{n+1}+C_{i, j+1}^{n+1}+C_{i, j-1}^{n}-2 C_{i, j}^{n}+C_{i, j+1}^{n}\right]}{2(\Delta Y)^{2}}$

Here the region of integration is considered as a rectangle with $X_{\max }=1$ and $Y_{\max }=14$ where $Y_{\max }$ corresponds to $Y=\infty$ which lies well outside both the momentum and thermal boundary layers. The maximum of $\mathrm{Y}$ was chosen as 14, after some preliminary numerical experiments such that the last two boundary conditions of (24) were satisfied within the tolerance limit $10^{-5}$. The mesh sizes have been fixed as $\Delta X=0.05, \Delta Y=0.05$ with time step $\Delta t=0.01$. The computations are executed initially by reducing the spatial mesh sizes by $50 \%$ in one direction, and later in both directions by $50 \%$. The results are compared. It is observed that, in all the cases, the results differ only in the fifth decimal place. Hence, the choice of the mesh sizes is verified as extremely efficient. The coefficients of $U_{i, j}^{n}$ and $V_{i, j}^{n}$ appearing in the finite difference equations are treated as constant at any onetime step. Here $i$ designates the grid point along the $\mathrm{X}$ direction, $j$ along the $\mathrm{Y}$-direction and $n$ in the time variable, $t$. The values of $\mathrm{U}, \mathrm{V}, \mathrm{T}$ and $\mathrm{C}$ are known at all grid points when $\mathrm{t}=0$ from the initial conditions.

The computations for $\mathrm{U}, \mathrm{V}, \mathrm{T}$ and $\mathrm{C}$ at a time level $(\mathrm{n}+$ 1 ), using the values at previous time level $\mathrm{k}$ are executed as follows. The finite-difference equation (34) at every internal nodal point on a particular $i-$ level constitutes a tri-diagonal system of equations and is solved by the robust Thomas algorithm as discussed in Carnahan et al. (1969). Thus, the values of $\mathrm{C}$ are known at every nodal point at a particular $i$ at $(\mathrm{n}+1)^{\text {th }}$ time level. Similarly, the values of $\mathrm{U}$ and $\mathrm{T}$ are calculated from equations (32), (33) respectively, and finally the values of $\mathrm{V}$ are calculated explicitly by using equation (31) at every nodal point on a particular $i$ - level at $(\mathrm{n}+1)^{\text {th }}$ time level. In a similar manner, computations are carried out by moving along $i$-direction. After computing values corresponding to each $i$ at a time level, the values at the next time level are determined in a similar manner. Computations are repeated until the steady state is attained. The steady state solution is assumed to have been reached when the absolute difference between the values of the velocity $\mathrm{U}$, temperature $\mathrm{T}$, as well as concentration $\mathrm{C}$ at two consecutive time steps are less than $10^{-5}$ at all grid points. The scheme is unconditionally stable. The local truncation error is $O\left(\Delta t^{2}+\Delta X^{2}+\Delta Y^{2}\right)$ and it tends to zero as $\Delta t, \Delta X$ and $\Delta Y$ tend to zero. It follows that 
the CNM scheme is compatible. Stability and compatibility ensure the convergence.

Since the present implicit Crank-Nicolson numerical difference code has been extensively validated against other numerical schemes by the authors Prasad et al. (2008), it is extremely reliable and we omit comparison solutions with previous studies here for brevity.

The derivatives involved in Eqs. (25) - (30) are evaluated using five-point approximation formula and then the integrals are evaluated using Newton-Cotes closed integration formula.

\section{RESUlTS AND DISCUSSION}

Extensive computations have been performed for the effects of the controlling thermofluid and hydrodynamic parameters on dimensionless velocity $(\mathrm{U})$, temperature $(\mathrm{T})$ and concentration $(\mathrm{C})$ and also on the local skin-friction $\left(\tau_{X}\right)$, average skin-friction $(\bar{\tau})$, local Nusselt number $\left(N u_{X}\right)$, average Nusselt number $(\overline{N u})$, local Sherwood number $\left(S h_{X}\right)$ and average Sherwood number $(\overline{S h})$. The present analysis concerns the case of optically thick boundary layers, where the thermal boundary layer is expected to become very thick as the medium is highly absorbing. The radiative diffusion (Rosseland model) adds a radiative conductivity to the conventional thermal conductivity. The effect of radiation is to thicken the thermal boundary layer similar to the effect of decreasing Prandtl number (the latter representing the ratio of viscous to thermal diffusion). Radiation supplements the thermal diffusion effectively enhancing the thermal diffusivity, as described by Siegel and Howell (1972).

In Fig. 3, the influence of radiation parameter, $\mathrm{N}$ on steady state velocity are shown. $N=\frac{k_{e} k}{4 \sigma_{s} T_{\infty}^{\prime 3}}$ and this defines the ratio of thermal contribution relative to radiation. For radiative heat transfer dominance in the boundary layer regime, $N \rightarrow 0$. For finite values of $\mathrm{F}$ there will be a simultaneous presence of thermal conduction and radiative transfer contributions. For $\mathrm{N}=1$ both modes will contribute equally. For $N \rightarrow \infty$ in Eq. (22), the term $\frac{4}{3} F \rightarrow 0$ and the energy equation reduces to the conventional unsteady conductionconvection equation i.e.

$$
\frac{\partial T}{\partial t}+U \frac{\partial T}{\partial X}+V \frac{\partial T}{\partial Y}=\frac{1}{\operatorname{Pr}} \frac{\partial^{2} T}{\partial Y^{2}}
$$

An increase in $\mathrm{N}$ from 3,5 to 10 , causes a significant decrease in velocity with distance into the boundary layer i.e. decelerates the flow. Velocities in all cases from the plate surface, peak close to the wall and then decay smoothly to zero in the free stream. We also note that with increasing values on $\mathrm{N}$ the time taken to attain the steady state is increased. Thermal radiation flux therefore has a de-stabilizing effect on the transient flow regime. This is important in polymeric and other industrial flow processes since it shows that the presence of thermal radiation while decreasing the temperatures, will affect flow control from plate surface into the boundary layer regime. For lower values of $\mathrm{Gr}=\mathrm{Gm}(=3)$, the time required to reach the steady state with $\mathrm{N}=3,5$ and 10 are 8.60, 9.44,9.65 respectively, where as for higher values of $\mathrm{Gr}=\mathrm{Gm}$ $(=5)$ the steady state is reached at 7.65,8.48 and 8.69 respectively which leads to conclude that increase in buoyancy force parameter $\mathrm{Gr}$ or $\mathrm{Gm}$ reduces the time to reach the steady state.

For various values of Schmidt number and Prandtl number, the transient velocity profiles are plotted in Fig. 4. Pr defines the ratio of momentum diffusivity $(v)$ to thermal diffusivity. It also expresses the ratio of the product of dynamic viscosity and specific heat capacity to thermal conductivity of the principal fluid. $\operatorname{Pr}<1$ physically implies that heat will diffuse faster than momentum in the fluid. For $\operatorname{Pr}=1$ the diffusion rates will be same for heat and momentum i.e. thermal and velocity boundary layer thickness will be equal. For Pr $>1$ momentum will diffuse faster that heat. An increase in Pr from 0.7 to 1.25 , clearly decrease significantly streamwise velocity, $\mathrm{U}$ for considerable distance into the boundary layer. Flow is therefore decelerated with the increase in Prandtl number. A close proximity to the plate surface, velocities are all maximized for any value of Prandtl number, and then descend gradually to zero far from the plate surface.

In Fig. 5, we have plotted the influence of surface concentration power law exponent $(\mathrm{m})$ and the surface temperature power law exponent (n) on velocity distribution with $\mathrm{Y}$ coordinate. For $\mathrm{n}=0$, the power law variation of concentration reduces from $q_{w}^{\prime}(x)=a x^{n}$ to $q_{w}^{\prime}(x)=a$ i.e. we obtain iso-species scenario (constant wall heat flux). Similarly for $\mathrm{m}=0$ the power law variation of temperature $q_{w}^{\prime}(x)=b x^{m}$ to $q_{w}^{\prime}(x)=b$ i.e we obtain constant wall heat flux scenario. For the doubly special case of $m=n=0$, we observe that the velocity is maximized throughout the boundary layer. With an increase in $\mathrm{m}$ to 0.5 , the velocity is reduced in the boundary layer. Further decrease is observed when $\mathrm{m}=\mathrm{n}=0.5$. As such increasing power law exponents in the plate surface concentration and temperature variations serve to decelerate the flow in the boundary layer. For $m=n=0$ the lowest time is achieved to arrive at the steady state. This value is increased for $\mathrm{m}=0$ and $\mathrm{n}=0.5$, but then reduced for $\mathrm{m}=\mathrm{n}=0.5$, although in the latter case it is still greater than for the classic case of $m=n=0$. To illustrate the effect of temperature exponent $\mathrm{m}$ and the radiation parameter $\mathrm{N}$ on the temperature, the transient temperature distribution near the plate at $\mathrm{X}=1.0$ is presented in Fig. 6. As expected, the temperature values are also significantly reduced with an increase in $\mathrm{N}$ as there is a progressive decrease in thermal radiation contribution accompanying this. All profiles are monotonic decays from the wall to the free stream. This trend concurs with the results of Beg et al. (1998, 2003), Chamkha et al. (2004) and Lin and Huang (1991), all these studies also employing the Rosseland-diffusion approximation. The maximum reduction in temperatures is witnessed relatively close to the plate surface since thermal radiation effects will be 
prominent closer the plate surface, rather into the free stream. With an increase in the power law temperature exponent $\mathrm{m}$ the temperature is reduced. It is also seen that, the time required to reach the steady state temperature is more at higher values of $N(=10)$ as compared to lower values of $\mathrm{N}(=5)$. Typical variations in the temperature profiles for different values of $\operatorname{Pr}$ and $\mathrm{N}$ are shown in Fig. 7. As expected, the numerical results show that an increase in the Prandtl number results in a decrease of the thermal boundary layer thickness and in general lower average temperature within the boundary layer. The reason is that smaller values of $\operatorname{Pr}$ is equivalent to increasing the thermal conductivity of the fluid, and therefore heat is able to diffuse away from the heated surface more rapidly than for higher values of Pr. Hence in the case of smaller Prandtl numbers the thermal boundary layer is thicker and the rate of heat transfer is reduced. From Figs. 1 and 4 it is obvious that an increase in the radiation parameter $\mathrm{N}$ results in decreasing velocity and temperature within the boundary layer, as well as decreased thickness of the velocity and temperature boundary layers. The transient concentration profiles are shown in Fig. 8 for different values of concentration exponent $\mathrm{n}$ and Sc. A rise in Sc strongly suppresses concentration levels in the boundary layer regime. All profiles decay monotonically from the plate surface (wall) to the free stream. Sc embodies the ratio of momentum diffusivity $(v)$ to molecular diffusivity (D). The concentration reduces with the increasing values of $\mathrm{n}$. As expected concentration is lower for systems with higher Sc. Time taken to reach the steady state increases as Sc increases from 0.6 to 0.94 .

The effect of buoyancy force parameter $\mathrm{Gr}$ or $\mathrm{Gm}$ on time to reach the steady state conditions are shown in Fig. 9. Concentration is boosted with an increase in $\mathrm{N}$ i.e. decrease in thermal radiation contribution. The parameter $\mathrm{N}$ does not arise in the species conservation equation (23) and therefore concentration filed is indirectly influenced by $\mathrm{N}$ via the coupling of the energy equation (22) with the momentum equation (21), the latter also being coupled with the convective acceleration terms in the species equation (23). However as with the response of the velocity and temperature fields, an increase in $\mathrm{N}$ decreases the time elapse to achieve the steady state. Therefore while greater thermal radiation augments diffusion of species in the regime it requires greater time to achieve the steady state i.e. when $\mathrm{Gr}=\mathrm{Gm}(=3)$ the time required to reach the steady state when $\mathrm{N}=3,5$ and 10 are 9.22, 9.36 and 9.52 where as for large $\mathrm{Gr}=\mathrm{Gm}(=5)$. The time required to reach the steady state are 9.03,9.24 and 9.26 respectively which leads to conclude that when the buoyancy force parameter $\mathrm{Gr}$ or $\mathrm{Gm}$ increases the time required to reach the steady state is reduced.

The effects of Sc, N, $\mathrm{n}$ and $\mathrm{m}$ on local skin friction is shown in Fig. 10. Increasing Sc clearly boosts the wall skin friction, $\tau_{X}$ which grows strongly from the leading edge to the downstream along the plate surface. Also with an increase in $\mathrm{N}$, acts to effectively accelerate the flow, concurring with trend in the computations for flat plate [Chamkha et al. (2001)] for radiationconvection boundary layers. The average values of skin-friction is shown in Fig. 11 as a function of time at $\mathrm{X}=1.0$ for various physical parameters. The average values of skin-friction decreases with an increase in $\mathrm{Sc}$ or $\mathrm{n}$ or $\mathrm{m}$ throughout the transient period. It is also observed that, average skin- friction increases as the radiation interaction parameter $\mathrm{N}$ increases.

The local Nusselt number $N u_{X}$ for different $\mathrm{n}, \mathrm{m}$ and $\mathrm{N}$ are shown in Fig. 12.The local heat transfer rate $N u_{X}$ increases with the increasing values of $\mathrm{N}$, heat transfer from the plate surface is therefore suppressed with increasing thermal radiation and enhanced with greater thermal conduction. Also $N u_{X}$ increases with the increase in $\mathrm{m}$ and decreases with the increasing $\mathrm{n}$. The average values of Nusselt number $\overline{N u}$ is shown in Fig. 13 as a function of time at $\mathrm{X}=1$.0.The average Nusselt number $\overline{\mathrm{Nu}}$ for different $\mathrm{n}, \mathrm{m}$ and $\mathrm{N}$ are shown in Fig. 11.The average Nusselt number increases with increasing $\mathrm{m}$ or $\mathrm{N}$. The effects of $\mathrm{m}, \mathrm{n}$ and $\mathrm{Sc}$ on the local and average Sherwood numbers are shown in Figs. 14 and 15 respectively. As expected surface species gradient i.e. mass transfer rate at the plate surface is strongly with a rise in Sc. Both the local and average Sherwood numbers increase with the increasing.

\section{CONCLUSIONS}

A detailed numerical study has been carried out for the effects of radiation past an impulsively started vertical plate with variable surface heat and mass flux. The dimensional less governing equations are solved by an implicit finite difference method of Crank-Nicolson type. The results are presented for major parameters including the radiation parameter $\mathrm{N}$, Prandtl number $\mathrm{Pr}$, thermal Grashof number Gr, mass Grashof number Gm, Schmidt number Sc, heat and mass flux exponent. A systematic study on the effects of various parameters on flow, heat and mass transfer characteristics is carried out. The particular conclusions drawn from the study can be listed as follows:

1. The time required to reach the steady state increases as radiation parameter increases.

2. The momentum boundary layer thickness decreases with the increase in Sc. The velocity decreases with the increase in $\mathrm{m}$ and $\mathrm{n}$. It is also observed that increase in Pr leads to a decrease in velocity. The time taken to reach the steady state increases as Pr and Sc increases.

3. Temperature decreases with the increase in $\mathrm{Pr}$ or the radiation parameter $\mathrm{N}$. Also increase in $\mathrm{m}$ leads to a decrease in temperature.

4. The local and average skin-friction decreases as $\mathrm{n}$ or $\mathrm{m}$ increases.

5. It also observed that local and average skinfriction increases as the radiation interaction parameter increases.

6. The local and average Nusselt number increases with the increase in the value of radiation parameter.

7. Both the local average Sherwood numbers increases with the increasing $\mathrm{n}$ or Sc. 


\section{REFERENCES}

Abd-El-Naby, M.A., M.E.E. Elbarbary and Y.N. Abdelazem (2003). Finite difference solution of radiation effects on MHD free convection flow over a vertical plate with variable surface temperature. J. Appl. Math 2, 65-86.

Beg, O.A., H.S. Takhar and M. Kumari (1998). Computational analysis of coupled radiation convection dissipative flow in a porous medium using the Keller-Box implicit difference scheme. Int. J. Energy Res. 22, 141-159.

Beg, O.A., H.S. Takhar, A.J. Chamkha, D. Filip and I. Pop (2003). Mixed radiation convection flow of an optically dense viscous fluid along a vertical surface in a non-Darcy geological porous system, Int. J. Applied Mechanics and Engineering 8(3), 483-496.

Bratis, J.C., J.R. Novotny (1974). Radiation-convection interaction in the boundary layer-regime of an enclosure. Int. J. Heat Mass Transfer 17, 365-379.

Carnahan, B., H.A. Luther and J.O. Wilkes (1969). Applied Numerical Methods. John Wiley and sons, New York.

Cess, R.D (1966). The interaction of thermal radiation with free convection heat transfer. Int. J. Heat Mass Transfer 9, 1269-1277.

Chamkha, A.J., H.S. Takhar and M. Soundalgekar (2001). Radiation effects on free convection flow past a semi-infinite vertical plate with mass transfer. Chem. Engg. J. 84, 335-342.

Chamkha, A.J., H.S. Takhar and O.A. Beg (2004). Radiative free convective non-Newtonian fluid flow past a wedge embedded in a porous medium. Int. J. Fluid Mech. Res. 31, 101-115.

Chang, C., K.T. Kang and J.R. Lloyd (1983). Radiation-natural convection interaction in twodimensional complex enclosure. ASME J. Heat Transfer 105, 89-95.

Cheng, E.H. and M.N. Ozisik (1972). Radiation with free convection in an absorbing, emitting and scattering medium. Int. J. Heat Mass Transfer 15, 1243-1252.

Das, U.N., R. Deka and V.M. Soundalgekar (1996). Radiation effects on flow past an impulsively started vertical plate-an exact solutions. J. Theo. Appl. Fluid Mech. 1 (2), 111-115.

Hadley, G.R. (1992). Transparent boundary condition for the beam propagation method. IEEE J. Quantum Electronics 28, 363-370.

Hossain, K.K. and K. Vafai (2001). The effect of radiation on free convection flow of fluid with variable viscosity from a porous vertical plate. Int. J. Thermal Sciences 40, 115-124.
Krishnaprakas, C.K., K.B. Narayana and P. Dutta (2001). Combined conduction and radiation heat transfer in a gray anisotropically scattering planar medium with diffuse-specular boundaries. Int. Comm. Heat and Mass Transfer 28, 77-86.

Lin, J.D. and J.M. Huang (1991). Radiation and convection in circular pipe with uniform wall heat flux. AIAA J. Thermophysics and Heat Transfer 5, 502-507.

Mansour, M.H. (1990). Radiative and free convection effects on the oscillatory flow past a vertical plate, Astrophysics and space science 166, 26-75.

Modest, M.F. (1993). Radiation Heat Transfer. MacGraw-Hill, New York.

Mukhopadhyay, S. (2009). Effects of radiation and variable fluid viscosity on flow and heat transfer along a symmetric wedge. J. Applied Fluid Mech. 2, 29-34

Muthucumaraswamy, R. and P. Ganesan (1998). Unsteady flow past an impulsively started vertical plate with heat and mass transfer. Heat and Mass transfer 34, 187-193.

Prasad, V.R., N.B. Reddy and R. Muthucumaraswamy (2006a). Finite difference analysis of radiation effects on MHD free convection flow with mass transfer past a semi-infinite vertical plate in the presence of heat source/sink. Int. Review of Pure and Applied Mathematics 2(2), 141-160.

Prasad, V.R., N.B. Reddy and R. Muthucumaraswamy (2006b). Transient radiative hydromagnetic free convection flow past an impulsively started vertical plate with uniform heat and mass flux. Theoretical Applied Mechanics 33(1),31-63.

Prasad, V.R., N.B. Reddy and R. Muthucumaraswamy (2007). Radiation and mass transfer effects on two-dimensional flow past an impulsively started infinite vertical plate. Int. J. Thermal Sciences 46(12), 1251-1258.

Prasad, V.R. and N.B. Reddy (2008). Radiation and mass transfer effects on an unsteady MHD convection flow past semi-infinite vertical plate permeable moving plate embedded in a porous medium with viscous dissipation. Indian J. Pure and Applied Physics 46, 81-92.

Ransom, J.B. and R.E. Fulton (1985). Concurrent implementation of the Crank-Nicolson method for heat transfer analysis. NASA Technical Memorandum 86448, Langley Research Center, Springfield, Virginia, USA.

Raptis, A. and C. Perdikis (1999). Radiation and free convection flow past a moving plate. Appl. Mech. Eng. 4, 817-821. 
Siegel, R. and J.R. Howell (1972). Thermal Radiation Heat Transfer, International student edition. MacGraw-Hill, New York.

Soundalgekar, V.M. (1977). Free convection effects on the Stokes problem for infinite vertical plate. ASME. J. Heat Transfer 99, 499-501.

Stewartson, K. (1951). On the impulsive motion of a flat plate in a viscous fluid. Quarterly Journal of Mechanics and Applied Mathematics 4, 182-198.

Wang, W. and G. Chen (2005). Heat and mass transfer model of dielectric-material-assisted microwave freeze-drying of skim milk with hygroscopic effect, Chemical Engineering Science 60(23), 6542 -6550 .

Vrentas, J.S. and C.M. Vrentas (1996). Axial moment analyses of convective heat and mass transfer processes. Chemical Engineering Science 51(6), 921-929.

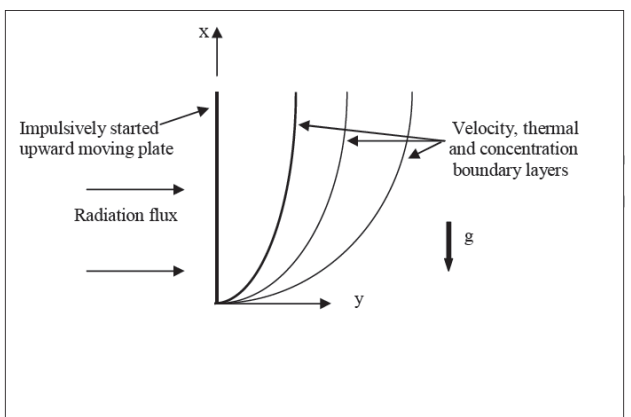

Fig.1. Physical model and coordinate system

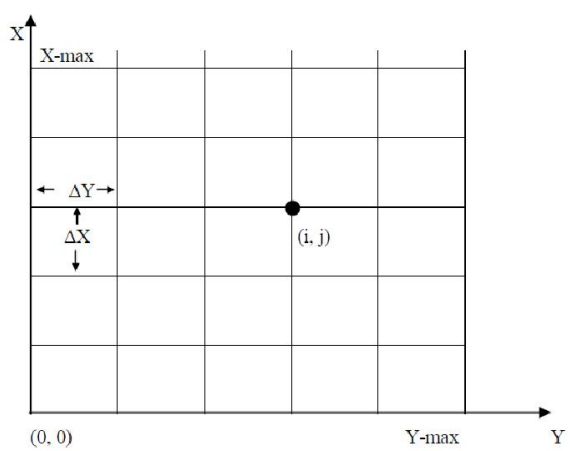

Fig.2. Grid System

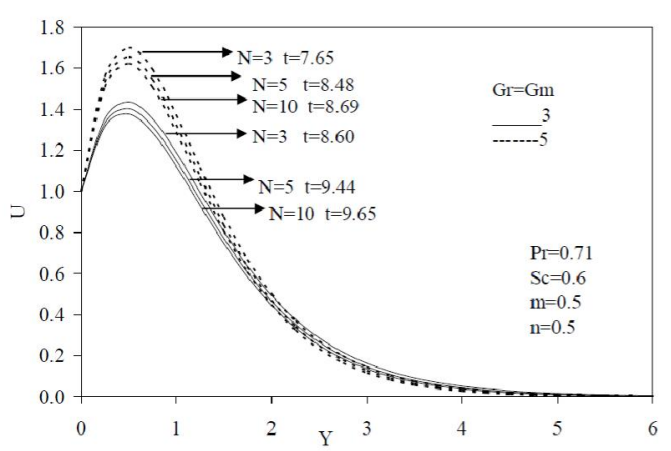

Fig.3. Velocity profiles at $\mathrm{X}=1.0$ for different $\mathrm{Gr}$, $\mathrm{Gm}, \mathrm{N}$.

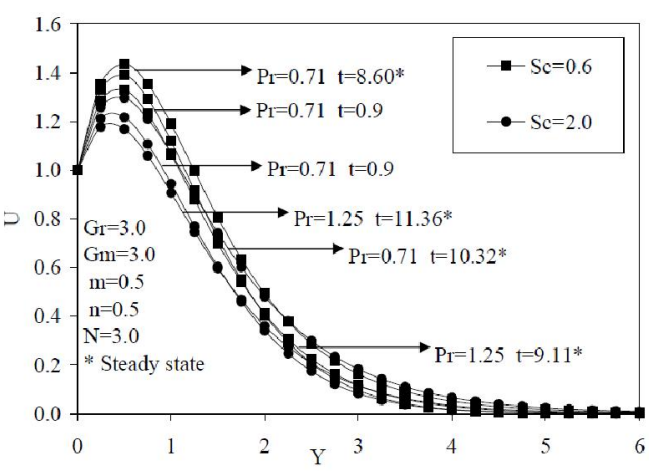

Fig. 4. Transient velocity profiles at $X=1.0$ for different Pr and Sc.

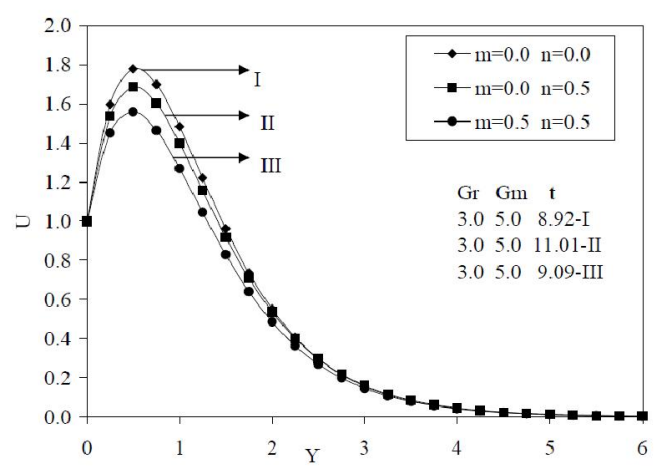

Fig. 5. Steady state velocity profiles at $X=1.0$ for different Gr, Gc, m, n.

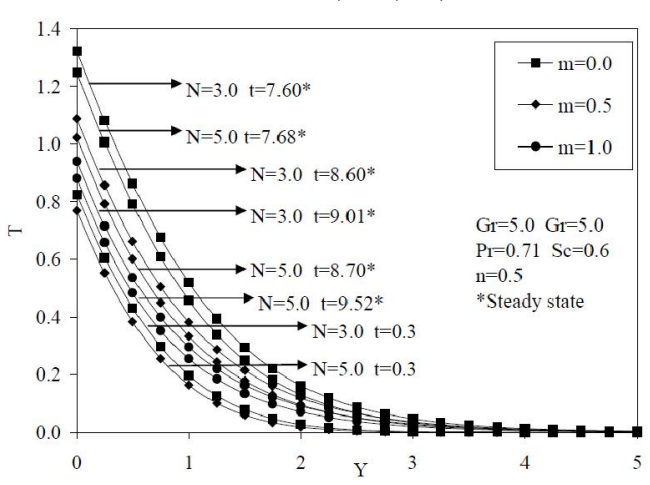

Fig. 6.Transient temperature profiles at $X=1.0$ for different $\mathrm{m}$ and $\mathrm{N}$.

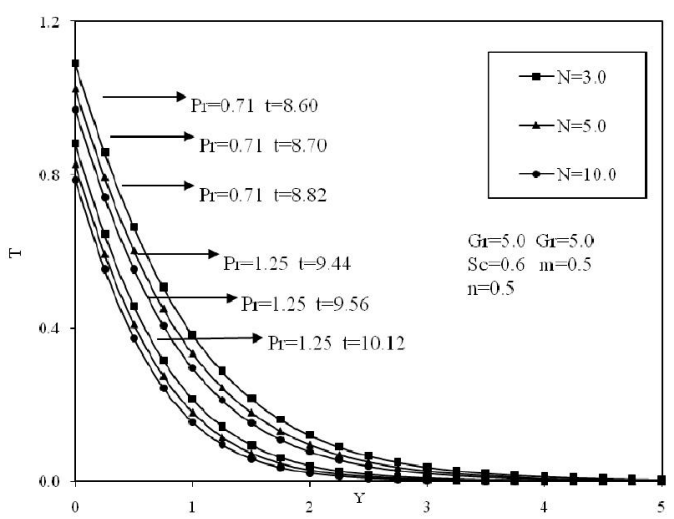

Fig. 7. Steady temperature profiles at $X=1.0$ for different Pr and N. 


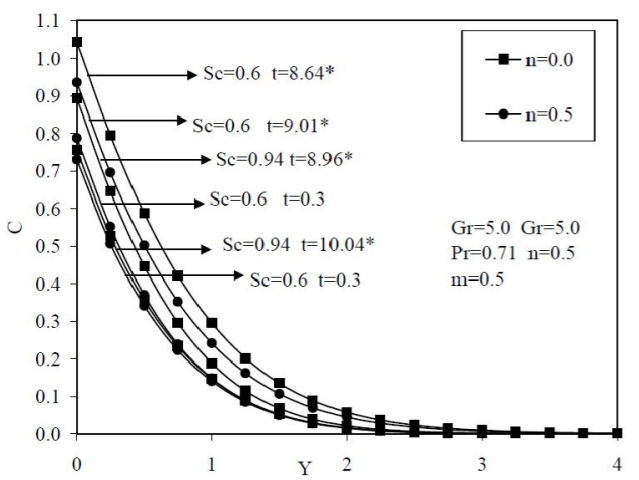

Fig. 8. Transient concentration profiles for different $\mathrm{n}$ and Sc.

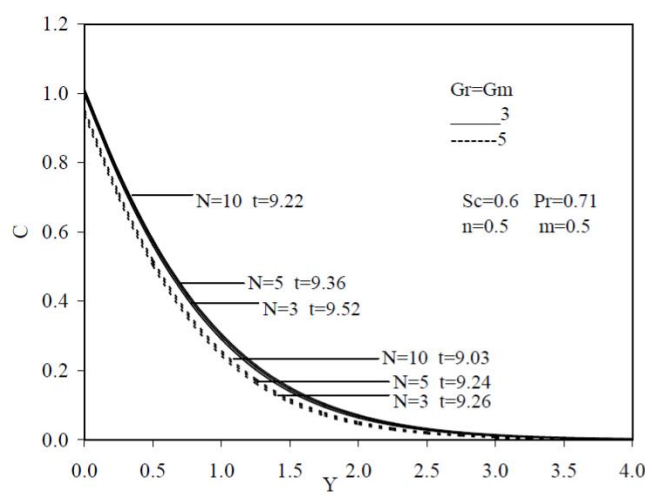

Fig. 9. Steady state concentration profiles at $\mathrm{X}=1.0$ for different $\mathrm{Gr}, \mathrm{Gm}, \mathrm{N}$.

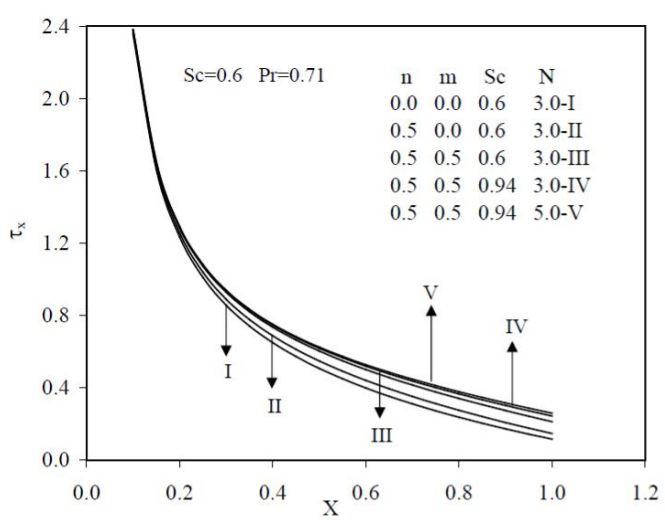

Fig. 10. Local skin friction.

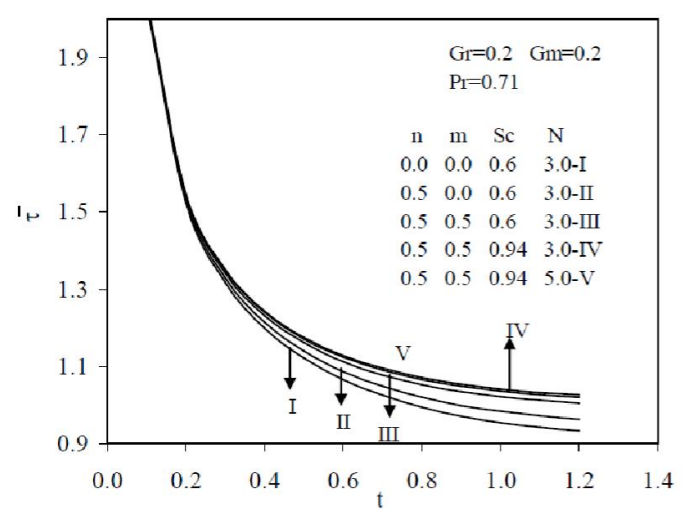

Fig. 11. Average skin friction.

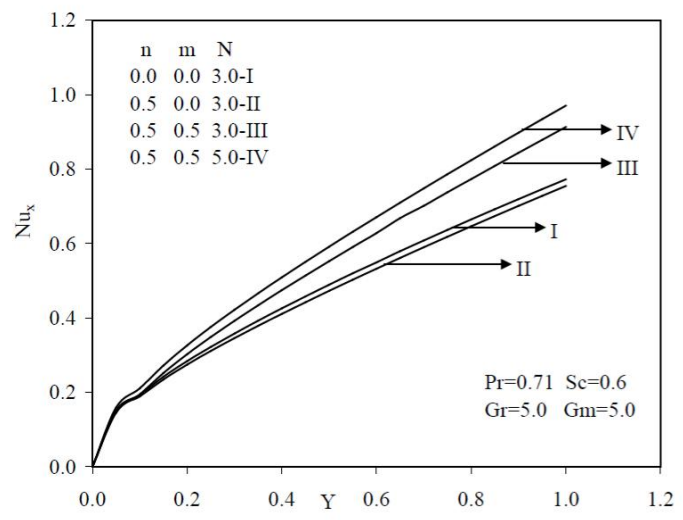

Fig. 12. Local Nusselt number.

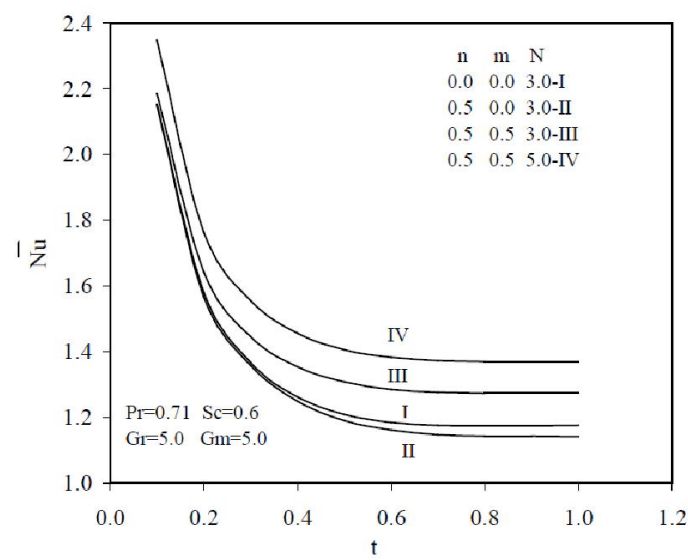

Fig. 13. Average Nusselt number.

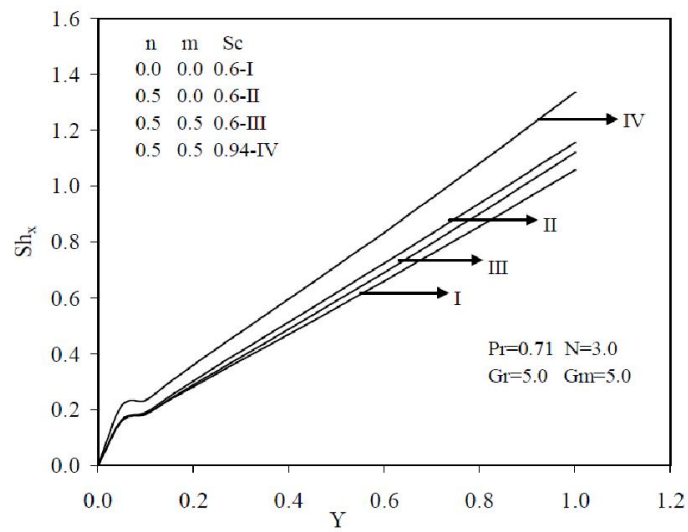

Fig.14. Local Sherwood number.

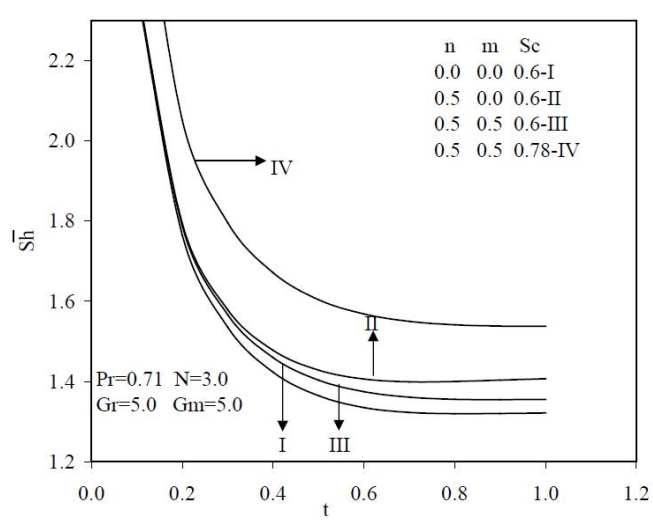

Fig. 15. Average Sherwood number. 